\title{
Intraoperative Hemodynamic Measurements of the Vertebral Artery and Common Carotid Artery
}

\author{
Yoshikazu OKAdA, Takeshi SHIMA*, Masahiro NishidA*, Kanji YAMAnE*, \\ Itsuo NAKAGAWA**, and Tomokatsu HORI \\ Department of Neurosurgery, Tokyo Women's Medical University, Tokyo; Departments of \\ ${ }^{*}$ Neurosurgery and ${ }^{* *}$ Anesthesiology, Chugoku Rousai Hospital, Kure, Hiroshima
}

\begin{abstract}
The hemodynamics in the vertebrobasilar artery (VBA) system were investigated in patients with vertebrobasilar insufficiency (VBI). Vertebral artery (VA) stump pressure and blood flows in the VA and common carotid artery (CCA) were intraoperatively measured in 45 patients who underwent surgical correction of the first segment of the VA $\left(\mathrm{V}_{1}\right)$ for angiographic tortuosity, kinking, and/or stenosis manifesting as symptomatic VBI. The effects of changes in the systemic arterial blood pressure (SABP) induced by trimethaphan, phenylephrine, and cervical epidural anesthesia were also investigated. The VA stump pressure was $79.3 \pm 13.6$ (mean \pm SD) $\mathbf{m m H g}$ and the ratio of the VA stump pressure to the SABP was $0.87 \pm 0.08$. The baseline values were SABP $90.5 \pm 10.1 \mathrm{mmHg}$, VA blood flow $53.4 \pm 33.0$ $\mathrm{ml} / \mathrm{min}$, and CCA blood flow $204.3 \pm 50.3 \mathrm{ml} / \mathrm{min}$. During changes in the SABP, autoregulation of the blood flow in the VA appeared tighter than in the CCA. During cervical epidural anesthesia, blood flows in both the VA and CCA were significantly reduced in response to SABP reduction. This study demonstrated that the VBA system maintains excellent autoregulation with good collateral flows and cervical sympathetic nerve function. However, this autoregulatory capacity may be overwhelmed by unexpected occlusion of the VA due to postural changes associated with tortuosity, kinking, and/or stenosis of the $V_{1}$ segment.
\end{abstract}

Key words: vertebral artery, carotid artery, autoregulation, sympathetic nerve

\section{Introduction}

Disturbances of the vertebrobasilar circulation may manifest as symptoms of vertebrobasilar insufficiency (VBI), such as vertigo, dizziness, visual disturbance, syncope, and drop attacks. ${ }^{2,3)}$ VBI may be caused by various lesions, including atherosclerotic narrowing and thromboembolism. ${ }^{17)}$ Vertebral artery (VA) blood flow may be disturbed by compressive occlusion of the VA by an extraluminal lesion associated with head movement. ${ }^{4-6,13,20,24,27)}$ However, the mechanism of vertebrobasilar circulatory disturbance caused by these lesions is unclear, even for compressive lesions of the cervical VA. Impaired autoregulatory response of the vertebrobasilar artery (VBA) system is another cause of VBI. ${ }^{25)}$ The autoregulatory response involves various factors such as brain metabolism and sympathetic nerve activity. The cervical sympathetic nerve has been implicated in the autoregulation of VBA blood

Received January 7, 2004; Accepted May 8, 2004 flow, but not definitely demonstrated in humans. There have been few reports of hemodynamic studies of the human VBA system, ${ }^{14,26)}$ due to the anatomical difficulties in approaching the VBA system, and the lack of appropriate methods to investigate the hemodynamics of the VBA system.

The present study simultaneously recorded the blood flows in the VA and common carotid artery (CCA) with electromagnetic flow meters during corrective surgery of the first segment of the VA $\left(V_{1}\right)$. The effects of changes in systemic arterial blood pressure (SABP) and sympathetic nerve blocking were also investigated.

\section{Materials and Methods}

Forty-five patients, 26 women and 19 men aged from 41 to 74 years (mean \pm SD $57 \pm 9$ years), presented with symptomatic VBI manifesting as vertigo and drop attacks. Conventional angiography showed serious kinking and stenosis by external compression of the $V_{1}$ segment (Fig. 1A). The symptoms were 

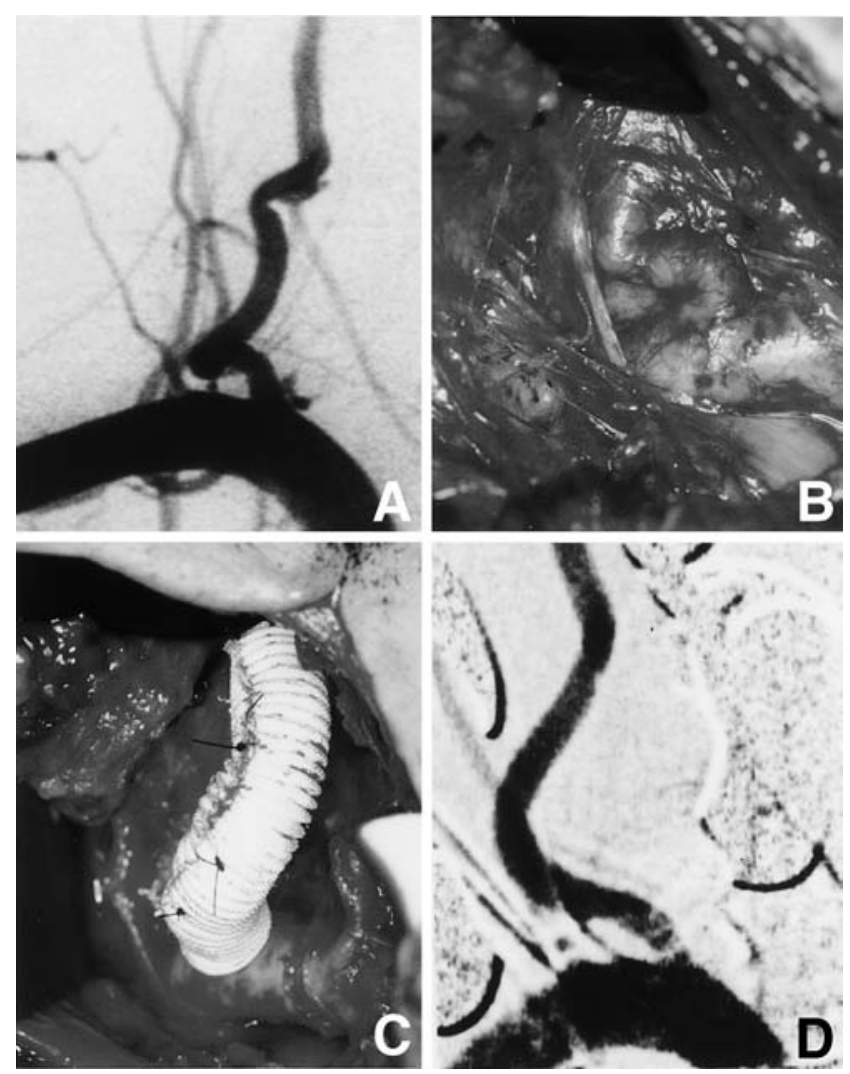

Fig. 1 (A) Preoperative vertebral angiogram showing serious tortuosity and kinking at the $V_{1}$ segment. (B) Intraoperative photograph showing serious kinking of the vertebral artery (VA) and sympathetic nerve bundles from the stellate ganglion. (C) Intraoperative photograph showing correction of the kinking of the VA by wrapping the involved VA with Dacron. (D) Intraoperative vertebral angiogram demonstrating relief of the serious tortuosity and stenosis at the $V_{1}$ segment.

frequently induced by postural changes such as extension and/or rotation of the neck and abrupt standing. All patients had received several courses of medical treatment over one year, which had not ameliorated their symptoms.

Informed consent concerning the surgical procedures and intraoperative hemodynamic investigations were obtained from the patients and their family before the surgery.

Surgery to correct the $\mathrm{V}_{1}$ segment was performed under general anesthesia induced by intravenous fentanyl 8 to $10 \mu \mathrm{g} / \mathrm{kg}$, pancuronium bromide $0.1 \mathrm{mg} / \mathrm{kg}$, and thiamylal sodium $2 \mathrm{mg} / \mathrm{kg}$, followed by intubation and maintenance with oxygen $3 \mathrm{l} / \mathrm{min}$ and nitrous oxide $3 \mathrm{l} / \mathrm{min}$. Cervical epidural anesthe- sia was also given at the request of some patients, since cervical epidural anesthesia was beneficial not only for intraoperative anesthesia but also for the postoperative relief of pain. The $\mathrm{V}_{1}$ segment was exposed from the subclavian artery to the sixth cervical level. The $V_{1}$ segment showed serious kinking or tortuosity caused by external compressive lesions in the surrounding connective tissue, fascia, and sympathetic nerve bundles (Fig. 1B). The surrounding connective tissue and fascia were removed and the sympathetic nerve bundles were carefully dissected from the involved $V_{1}$ segment. The $V_{1}$ segment was wrapped with a prosthesis (Dacron) to reconstruct a smooth curved course to the distal VA (Fig. 1C, D).

The hemodynamic studies were started when the ipsilateral VA was first exposed during surgery. The distal $V_{1}$ segment was punctured with a 26gauge needle to record the VA stump pressure by cross-clamping the proximal $\mathrm{V}_{1}$ segment. The blood flows in the VA and CCA were measured with electromagnetic flow meters (MFV 1100; Nihon Kohden, Tokyo). The radial artery was cannulated to measure the SABP and to sample blood. Respiration was controlled mechanically to maintain normoxia and normocapnia, with $\mathrm{PCO}_{2}$ of 35 to $45 \mathrm{mmHg}$. We prepared 3 or $3.5 \mathrm{~mm}$ probes for the VA and 6 or $7 \mathrm{~mm}$ probes for the CCA. Hypotension and hypertension were introduced by the intravenous injection of trimethaphan camsylate $(1 \mathrm{mg} / \mathrm{min})$ and phenylephrine (0.1 to $0.2 \mathrm{mg}$ ), respectively. The dose used was intended to decrease or increase the SABP by less than $30 \%$ of the baseline value.

Prior to the induction of general anesthesia, an epidural tube was inserted at the C7-T1 intervertebral space. Epidural anesthesia was confirmed with a test injection of $1 \%$ mepivacaine $(10 \mathrm{ml})$ to cause hypesthesia, hypotension, and Horner's sign. At least 2 hours later, an intraoperative injection of $1 \%$ mepivacaine $(10 \mathrm{ml})$ was administered, and the SABP, and blood flows in the VA and CCA were monitored for 10-15 minutes.

The hemodynamic studies were performed in the following order: baseline, induced hypotension, induced hypertension, and epidural anesthesia. SABP, and blood flows in the VA and CCA were measured when the maximum change of the SABP was observed within 10 minutes following drug administration. The parameters were observed for at least 15 minutes after drug administration.

Statistical analyses were performed with the paired t-test and statistical significance was determined as $\mathrm{p}<0.05$. All values shown are mean \pm SD. 

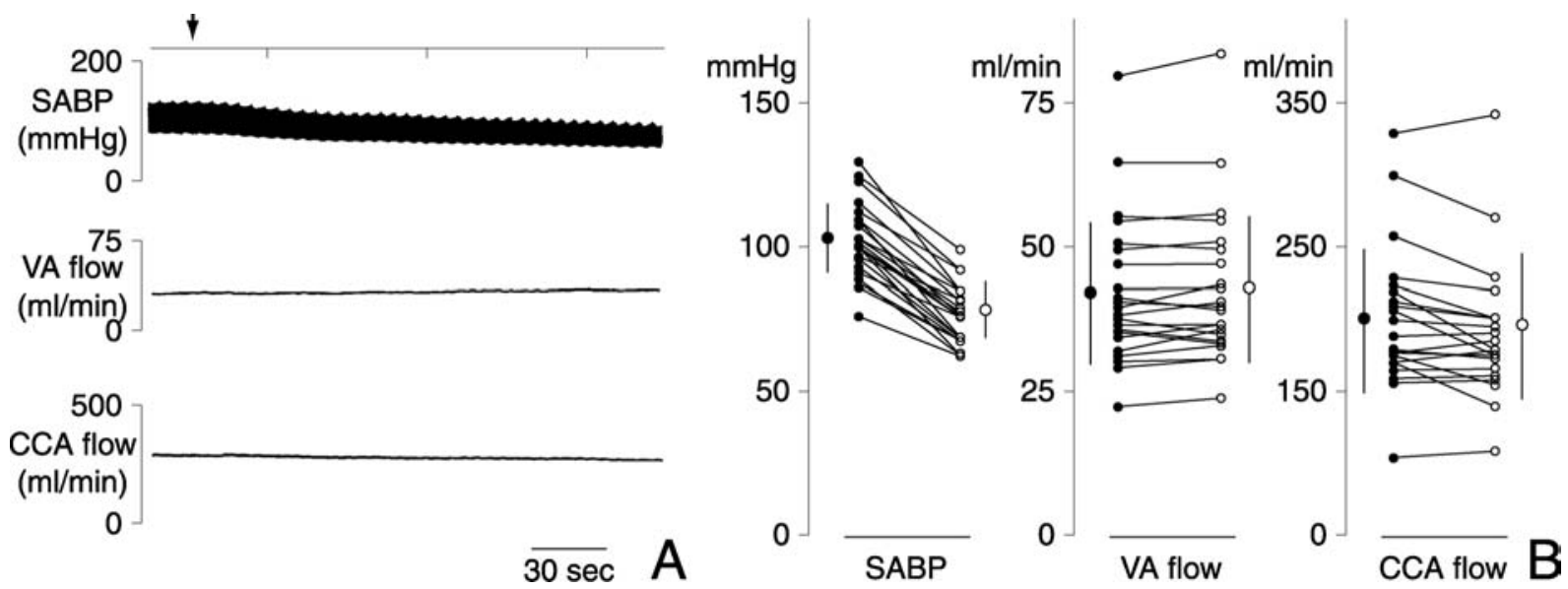

Fig. 2 (A) Representative recording of the effects of induced hypotension (arrow) on the systemic arterial blood pressure (SABP), and blood flows in the vertebral artery (VA) and common carotid artery (CCA). Despite evident decreases in the SABP, the VA blood flow is constant, and the CCA blood flow shows very mild reduction. (B) Summary of the SABP, and VA and CCA blood flows during induced hypotension in 22 patients. Closed circles and open circles indicate before and after induced hypotension, respectively. The larger closed and open circles with vertical bars indicate the mean \pm SD of the SABP, and VA and CCA blood flows before and after induced hypotension, respectively.

\section{Results}

The hemodynamic studies were performed without complications. The mean VA stump pressure ranged from 55 to $108 \mathrm{mmHg}(79.3 \pm 13.6 \mathrm{mmHg}, \mathrm{n}=19)$. The mean SABP was $90.5 \pm 10.1 \mathrm{mmHg}$. The ratio of mean VA stump pressure to mean SABP was 0.87 $\pm 0.08$

The baseline SABP, and blood flows in the VA and CCA were measured in 45 patients. The blood flow in the VA varied widely in each patient, ranging from 24 to $170 \mathrm{ml} / \mathrm{min}$. The baseline $\mathrm{SABP}$, and blood flows in the VA and CCA were 97.6 \pm 15.0 $\mathrm{mmHg}, \quad 53.4 \pm 33.0 \mathrm{ml} / \mathrm{min}, \quad$ and $\quad 204.3 \pm 50.3$ $\mathrm{ml} / \mathrm{min}$, respectively. During these measurements, arterial blood gas analysis revealed $\mathrm{pH}, \mathrm{PaCO}_{2}$, and $\mathrm{PaO}_{2}$ of $7.416 \pm 0.053,37.4 \pm 3.9 \mathrm{mmHg}$, and 177.2 $\pm 48.3 \mathrm{mmHg}$, respectively.

Mild hypotension and hypertension were smoothly induced within a few minutes of drug administration in all patients. A representative recording demonstrates the effect of induced hypotension on the SABP, and blood flows in the VA and CCA (Fig. 2A). Administration of trimethaphan induced a significant decrease in SABP from $103.7 \pm 13.2$ to $78.7 \pm 10.6 \mathrm{mmHg}(\mathrm{p}<0.0001, \mathrm{n}=22)$, no significant change in blood flow in the VA from $42.5 \pm$ 13.2 to $42.9 \pm 13.3 \mathrm{ml} / \mathrm{min}$ ( $\mathrm{p}<0.332$ ), and a significant decrease in blood flow in the CCA from $203.0 \pm 51.6$ to $192 \pm 49.0 \mathrm{ml} / \mathrm{min}(\mathrm{p}<0.01)$ (Fig. 2B).
A representative recording following phenylephrine administration demonstrates the strict autoregulatory capacity of blood flows in both the VA and CCA following induced hypertension (Fig. 3A). Phenylephrine administration produced an increase in SABP from $84.6 \pm 14.3$ to $122.5 \pm$ $16.3 \mathrm{mmHg}(\mathrm{p}<0.0001, \mathrm{n}=23$ ), but no significant change in blood flow in the VA from $43.7 \pm 13.7$ to $44.3 \pm 13.7 \mathrm{ml} / \mathrm{min}$, and in the CCA from $198.3 \pm$ 51.6 to $200.0 \pm 43.8 \mathrm{ml} / \mathrm{min}(\mathrm{p}<0.714, \mathrm{n}=21)$ (Fig. 3B).

A representative recording demonstrates the effects of cervical epidural anesthesia on SABP, and blood flows in the VA and CCA (Fig. 4A). Blood flows in both the VA and CCA progressively declined in response to the decrease in SABP. The effects of epidural anesthesia were evaluated 10 to 15 minutes after the epidural administration of mepivacaine. Cervical epidural anesthesia induced a significant decrease in SABP from $103 \pm 18.7$ to $79.0 \pm 11.3 \mathrm{mmHg}$ within 15 minutes $(\mathrm{p}<0.0001$, $\mathrm{n}=17$ ), and significant reductions in the blood flow in the VA from $43.4 \pm 10.3$ to $36.4 \pm 10.7 \mathrm{ml} / \mathrm{min}$ $(\mathrm{p}<0.0001, \mathrm{n}=17)$ and in the CCA from $194.2 \pm$ 48.0 to $180 \pm 52.6 \mathrm{ml} / \mathrm{min} \quad(\mathrm{p}<0.0216, \mathrm{n}=17)$ (Fig. 4B).

The relationships were studied between quantitative changes in SABP and blood flows in the VA and CCA in response to induced hypotension, hypertension, and cervical epidural anesthesia (Fig. 5). Induced hypotension caused wide variability in 

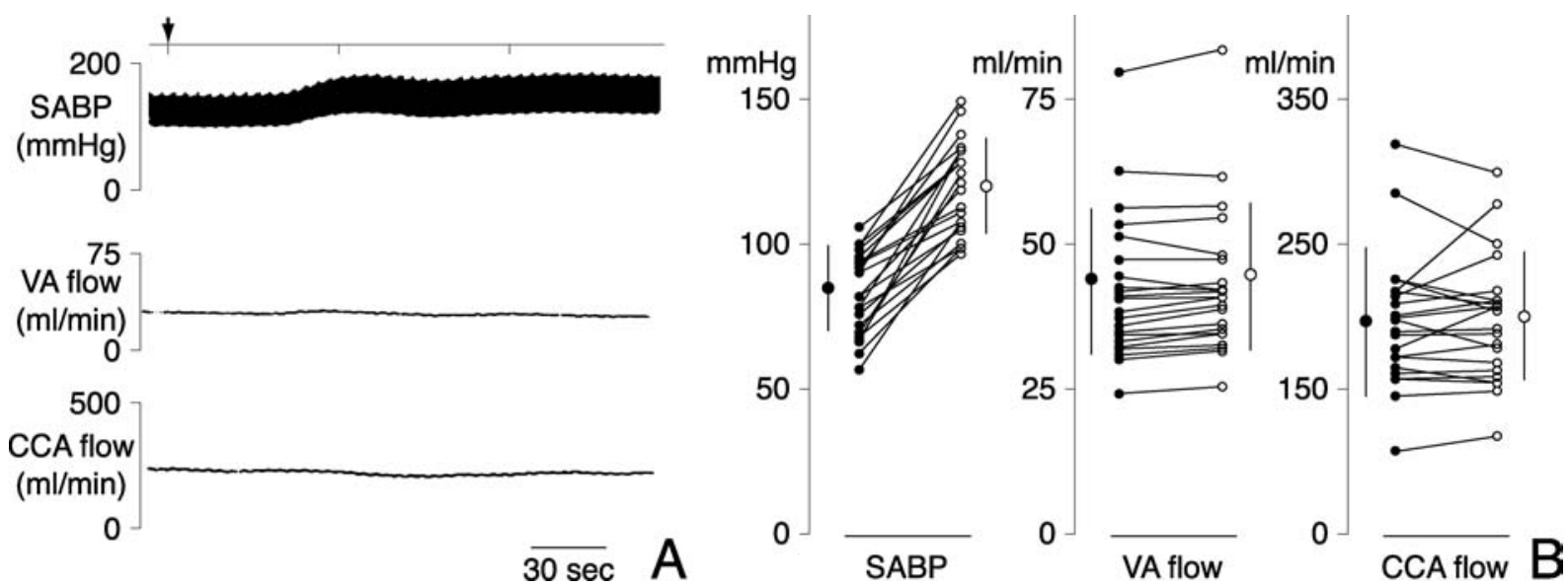

Fig. 3 (A) Representative effect of induced hypertension (arrow) on systemic arterial blood pressure (SABP), and blood flows in the vertebral artery (VA) and common carotid artery (CCA). (B) Summary of SABP, and VA and CCA blood flows during induced hypertension in 23 patients. Closed circles and open circles indicate before and after induced hypertension, respectively. The larger closed and open circles with vertical bars indicate the mean \pm SD of the SABP, VA and CCA blood flows before and after induced hypertension, respectively.

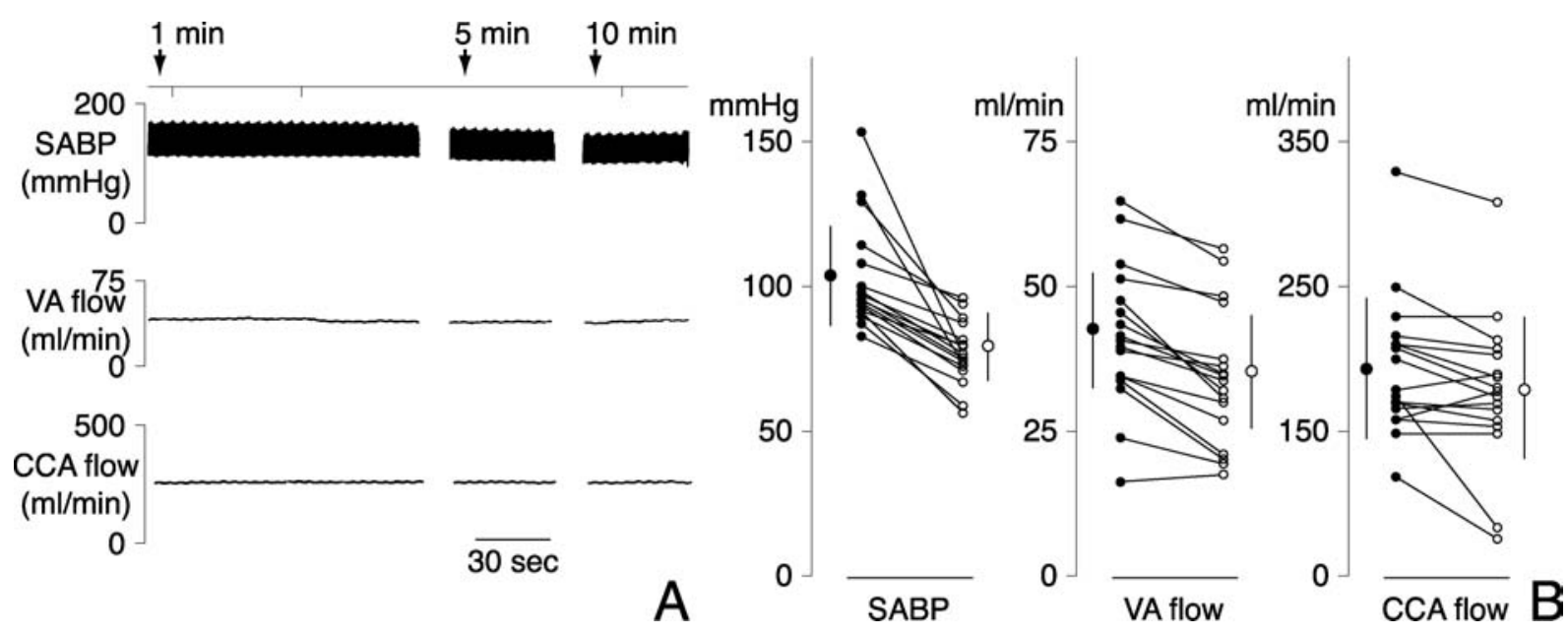

Fig. 4 (A) Representative recording of the effect of cervical epidural anesthesia (arrows) on systemic arterial blood pressure (SABP), and vertebral artery (VA) and common carotid artery (CCA) blood flows. A progressive decrease in SABP after epidural injection of mepivacaine resulted in concomitant decline of the blood flows in the VA and CCA. (B) Summary of the effects of cervical epidural anesthesia on SABP, VA and CCA blood flows. Closed circles and open circles indicate before and after epidural anesthesia, respectively. The larger closed and open circles with vertical bars indicate the mean \pm SD of the SABP, and VA and CCA blood flows before and after anesthesia, respectively.

SABP, blood flow in the VA remained constant, whereas that in the CCA decreased. There was no significant correlation between blood flow in either the VA or the CCA and the SABP during induced hypotension. Induced hypertension caused a significant increase in SABP, but blood flows in the VA and CCA maintained tight autoregulation, so no significant correlation was observed. Epidural anesthesia impaired autoregulation of blood flows in both the VA and CCA, so strong correlations were found between SABP and blood flows in the VA $\left(\mathrm{r}^{2}=0.50, \mathrm{p}<0.002\right)$ and CCA $\left(\mathrm{r}^{2}=0.563, \mathrm{p}<\right.$ $0.001)$.

Surgical complications were seen in three patients: One patient had lymphorrhea due to ruptured lymph vessels, and two patients had Horner's 

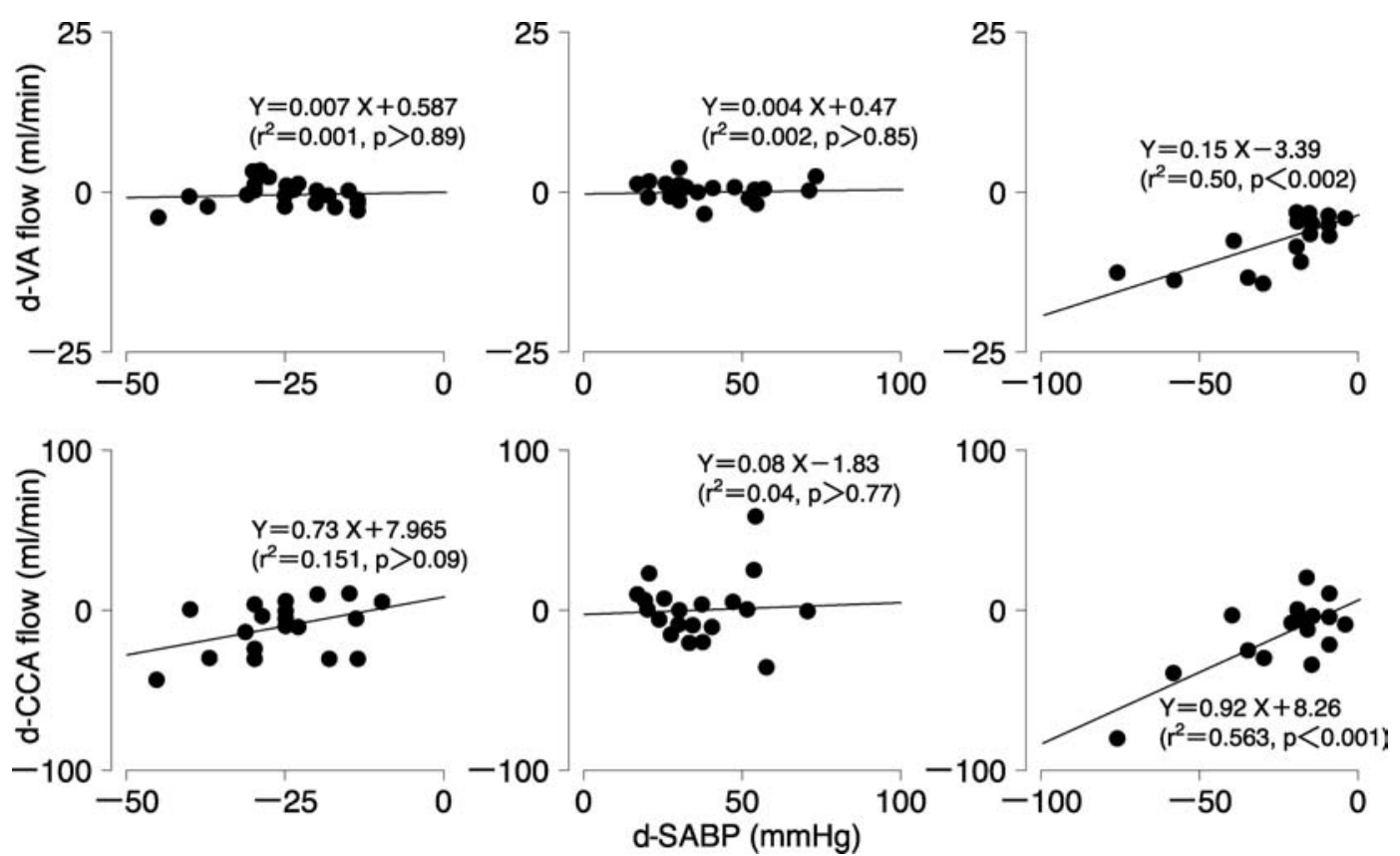

Fig. 5 Summary of the relationships between the systemic arterial blood pressure (SABP) and blood flows in the vertebral artery (VA) and common carotid artery (CCA) 10 to 15 minutes after induced hypotension (left column), hypertension (center column), and cervical epidural anesthesia (right column). d-SABP, d-VA flow, and d-CCA flow are the differences in values before and after induced hypotension, induced hypertension, and cervical epidural anesthesia.

syndrome due to cervical sympathetic nerve injuries. One patient with lymphorrhea was reoperated and completely cured, but Horner's syndrome persisted in the other at 12 months after the surgery. Clinical outcomes were evaluated at 6 to 12 months after the surgery. Forty patients were completely relieved of VBI symptoms. Some mild symptoms such as dizziness persisted in the other five patients.

\section{Discussion}

This study demonstrated that the blood flow in the VA is tightly maintained during induced hypertension and hypotension. Furthermore, the sympathetic nervous system is important in the autoregulatory response to control the blood flows in both the VA and CCA.

Stump pressure of the internal carotid artery (ICA) has been measured in many patients undergoing carotid surgery, but stump pressure of the VA has not been well documented. Some studies of ICA stump pressure have indicated a critical threshold, 25 to $50 \mathrm{mmHg}$, below which cerebral ischemia occurs. ${ }^{1,15)}$ In our carotid endarterectomy study, the ICA stump pressure was $52 \pm 17 \mathrm{mmHg}$ and the ratio of ICA stump pressure to SABP was 0.52 , which were significantly lower than the VA stump pressure of $79.3 \pm 13.6 \mathrm{mmHg}$ and the ratio of VA stump pressure to SABP of $0.87 .{ }^{18)}$ Clinically, unilateral interruption of blood flow in the VA is rarely associated with ischemic symptoms in the vertebrobasilar territory. The size of the contralateral VA and the configuration of the circle of Willis influence the likelihood of symptomatic unilateral occlusion. Review of 100 cases of VA ligation revealed that VA ligation was far less hazardous than ligation of the carotid artery, as long as the opposite VA was functional. ${ }^{23)}$ The same finding was established after temporary VA occlusion, as subjective symptoms were not manifested by patients with a normal configuration of the circle of Willis. ${ }^{16)}$ These findings suggest that the VA receives good collateral flow via the contralateral VA and the posterior communicating arteries.

Cerebral autoregulation attempts to maintain constant blood flow during changes in perfusion pressure, ${ }^{12)}$ but autoregulation of the VBA system has not been investigated in detail. The effects of induced hypertension and hypotension on both ICA and VA blood flows were investigated by reversible clamping of the monkey aorta and measurement with electromagnetic flow meters, findings that the efficiency of autoregulation of VA blood flow was superior to that of ICA blood flow. ${ }^{28)}$ In contrast, 
comparison of the autoregulatory capacity of the carotid artery and VA blood flow in rhesus monkeys with the photoelectric method demonstrated that carotid blood flow remained constant even when the blood pressure was reduced to $60 \mathrm{mmHg}$, whereas VA blood flow began to decrease at SABP as high as $70 \mathrm{mmHg}{ }^{21)}$ Therefore, autoregulation of VA blood flow is less effective than that of CCA blood flow. In our study, excellent autoregulatory responses were observed for both VA and CCA blood flows, although VA blood flow may have been better preserved during hypotension.

The involvement of the sympathetic nervous system in regulation of cerebral circulation remains controversial. ${ }^{8-11,22,28)}$ Some experimental and clinical studies have reported increases in cerebral blood flow (CBF) following cervical sympathectomy, ${ }^{10,22)}$ but many other studies showed no significant changes in CBF following either sympathectomy or sympathetic stimulation..$^{8,9,11)}$ These inconsistent results of neural regulation of $\mathrm{CBF}$ may be due to species differences; differences in sites of CBF measurement; differences in the method and the timing of measurement of CBF; and differences in the stimulation or blocking of the sympathetic nerve. Clinical impairment of cerebral autoregulation occurred in three patients with Shy-Drager syndrome, in which loss of sympathetic and parasympathetic reflexes was correlated with impaired cerebral autoregulation. ${ }^{14)}$ Consequently, CBF passively followed the changes in arterial perfusion pressure caused by induced hypertension or hypotension. The present study of the effects of cervical epidural anesthesia showed that blood flows in the VA and CCA were reduced following changes in SABP. This response may be mainly due to blocking of the cervical sympathetic nerve and partly due to diminished cardiac output caused by epidural anesthesia. Therefore, we suggest that the cervical sympathetic nervous system is involved in the regulation of the human VA and CCA blood flows. In our study, the differential effects of induced hypotension by ganglioblocking drugs versus cervical epidural anesthesia may be accounted for by the completeness of cervical sympathetic nerve blocking. Cervical epidural anesthesia may produce more complete blockade of the cervical sympathetic nerve than systemic administration of trimethaphan to induce mild hypotension. Therefore, the cervical sympathetic nerves may still be functionally intact during induced hypotension by trimethaphan.

Patients with VBI present with vertigo, dizziness, and visual disturbances. ${ }^{2,3)}$ Various etiologies have been proposed for this disease including throm- boembolic events and intermittent obstruction of the VA by extrinsic compressive lesions at the cervical level. The two main mechanisms causing VBI through external compression of the VA are sympathetic irritation and intermittent occlusion of the VA. ${ }^{19)}$ The VA may be occluded by compression at the median border by the anterior scalenus muscle and the thyro-cervical trunk. ${ }^{19)}$ Fifteen cases of intermittent rotational symptoms were caused by fascia compression enhanced by kinking of the VA. ${ }^{7)}$ Such dynamic VA occlusion is more likely to be symptomatic if contralateral flow is insufficient due to agenesis of the VA. In our patients, good collateral potential and excellent autoregulatory response were observed in the VBA system. The cervical sympathetic nerve was apparently important in the autoregulatory responses. Therefore, the pathogenesis of VBI in patients with serious kinking and tortuosity at the $\mathrm{V}_{1}$ segment may occur because postural and positional changes act through external compressive lesions to reduce the perfusion pressure of the VA, which exceeds the autoregulatory capacity of the VA blood flow. Consequently, surgical correction of the VA affected by external compressive lesions can relieve transient ischemic attacks of the VBA system.

\section{References}

1) Archie JP, Feldtman RW: Determinants of cerebral perfusion pressure during carotid endarterectomy. Arch Surg 117: 319-322, 1982

2) Bradshaw P, McQuaid P: The syndrome of vertebrobasilar insufficiency. Q J Med 32: 279-296, 1963

3) Cartlidge NEF, Whisnant JP, Elveback LR: Carotid and vertebral basilar transient cerebral ischemic attacks: a community study. Mayo Clin Proc 52: 117-120, 1977

4) George B, Laurian C: Pathophysiology, in George B, Laurian C (eds): The Vertebral Artery; Pathology and Surgery. Wien, New York, Springer-Verlag, 1987, pp 102-113

5) George B, Laurian C: Impairment of vertebral artery flow caused by extrinsic lesions. Neurosurgery 24: 206-214, 1989

6) Grad A, Baloh RW: Vertigo of vascular origin. Clinical and electronystagmographic features in 84 cases. Arch Neurol 46: 281-284, 1989

7) Hadrin CA, Poser CM: Rotational obstruction of the vertebral artery due to redundancy and extralaminal cervical fascial bands. Ann Surg 158: 133-137, 1963

8) Heistad DD, Marcus ML: Evidence that neural mechanism do not have important effects on cerebral blood flow. Circ Res 42: 295-302, 1978

9) Heistad DD, Marcus ML, Gross PM: Effects of sympathetic nerves on cerebral vessels in dog, cat, and monkey. Am J Physiol 234: H544-H552, 1978 
10) James IM, Miller RA, Purves MJ: Observations on the extrinsic neural control of cerebral blood flow in the baboon. Circ Res 25: 77-93, 1969

11) Kuwabara S, Ishikawa S, Shima T, Miyazaki $M$, Uozumi T: [Effects of electric stimulation of the superior cervical and steallate ganglia on basilar arterial flow]. No To Shinkei 31: 421-429, 1979 (Jpn, with Eng abstract)

12) Lassen NA: Cerebral blood flow and oxygen consumption in man. Physiol Rev 39: 183-238, 1959

13) Mapstone $T$, Spetzler RF: Vertebrobasilar insufficiency secondary to vertebral occlusion from a fibrous band. J Neurosurg 56: 581-583, 1982

14) Meyer JS, Shimazu K, Fukuuchi Y, Ohuchi T, Okamoto S, Koto A, Ericsson D: Cerebral dysautoregulation in central neurogenic orthostatic hypotension (Shy-Drager syndrome). Neurology 23: 262-273, 1973

15) Moore WS, Yee JM, Hall AD: Collateral cerebral blood pressure: An index to tolerance to temporary carotid occlusion. Arch Surg 106: 520-523, 1973

16) Nagashima C, Iwama K, Sakata E, Miki Y: Effects of temporary occlusion of a vertebral artery on the human vestibular system. J Neurosurg 33: 388-394, 1970

17) Naritomi H, Sakai F, Meyer JS: Pathogenesis of transient ischemic attacks within the vertebrobasilar arterial system. Arch Neurol 36: 121-128, 1979

18) Okada Y, Shima T, Matsumura S, Nishida $M$, Tamada T, Okita S: [Intraoperative monitoring of cerebral function and carotid hemodynamics during carotid endarterectomy]. No Shinkei Geka 17: 925-931, 1989 (Jpn, with Eng abstract)

19) Powers SR, Drislane TM, Nevins S: Intermittent vertebral artery compression: a new syndrome. Surgery 49: 257-264, 1961

20) Sakai F, Ishii K, Igarashi H, Suzuki S, Kitani N, Kanda T, Tazaki Y: Regional cerebral blood flow during an attack of vertebrobasilar insufficiency. Stroke 19: 1426-1430, 1988

21) Sato T: [Comparison of the efficiency in autoregulation of CBF in the territory supplied by carotid and vertebral arterial system of rhesus monkey]. Jpn J Stroke 2: 240-245, 1980 (Jpn)

22) Shenkin HA: Cervical sympathectomy on patients with occlusive cerebrovascular disease. Arch Surg 98: 317-320, 1969

23) Shintani A, Zervas N: Consequence of ligation of the vertebral artery. J Neurosurg 36: 447-450, 1972

24) Spetzler RF, Hadley MN, Martin NA, Hopkins LN, Carter P, Bundny J: Vertebrobasilar insufficiency. Part 1: Microsurgical treatment of extracranial vertebrobasilar disease. J Neurosurg 66: 648-661, 1987

25) Strandgaard S, Paulson OB: Cerebral autoregulation. Stroke 15: 413-416, 1984

26) Taki W, Handa H, Higa T, Tanada S, Fukuyama H, Fujita T, Yonekawa Y, Kameyama M, Torizuka K: Distribution of the blood flow supplied by the vertebral artery in humans as assessed by emission CT. Stroke 15: 469-474, 1984

27) Troost BT: Dizziness and vertigo in vertebrobasilar disease. Part II. Central causes and vertebrobasilar disease. Stroke 11: 413-415, 1980

28) Yoshida K, Meyer JS, Sakamoto K, Handa J: Autoregulation of cerebral blood flow. Electromagnetic flow measurements during acute hypertension in the monkey. Circ Res 19: 726-738, 1966

Address reprint requests to: Y. Okada, M.D., Department of Neurosurgery, Tokyo Women's Medical University, 8-1 Kawada-cho, Shinjuku-ku, Tokyo 162-8666, Japan.

e-mail: yokada@nij.twmu.ac.jp 